\title{
Texture and Phase Analysis in Nanocrystalline Ni Thin Films by Precession Electron Diffraction Microscopy
}

\author{
Szu-Tung $\mathrm{Hu}^{1}$, Lauren Morganti ${ }^{1}$, Shreyas Rajasekhara ${ }^{2 *}$, Khalid Hattar ${ }^{2}$, Paulo Ferreira ${ }^{1}$ \\ ${ }^{1}$ Materials Science and Engineering, University of Texas at Austin, Austin TX, USA \\ ${ }^{2}$ Sandia National Laboratories, Albuquerque, NM, USA \\ * Now at Intel, Hillsboro, OR, USA
}

Grain refinement is a powerful method to obtain high strength in metals and alloys. However, in materials with nano grain sizes, rapid grain growth may occur due to the high curvature of grain boundaries In addition, as grain growth occurs, the local texture may evolve, which affects the mechanical properties. Therefore, to achieve nanocrystalline metal films with both high strength and ductility it is imperative to correlate the film thickness with grain size and local texture as a function of annealing.

In this work, nanocrystalline Ni thin films with thicknesses of $30 \mathrm{~nm}$ and $120 \mathrm{~nm}$ were subjected to an annealing treatment at $350 \mathrm{C}$ for $20-80$ minutes. To identify the average grain size, grain orientation and phase fraction, precession electron diffraction was used. However, the reliability of the precession analysis is sometimes low due to the contribution of various grains to the diffraction pattern (DP). To improve the analysis, a two-step method was used where 1) the DP is filtered for noise threshold, spot enhance loop, gamma, spot radius and softening loop [1], and 2) the indexed DP is subjected to a reliability threshold of $15 \%$. In this fashion, the reliability was improved by $5-10 \%$.

The results show the presence of fcc and hcp phases (Fig. 1) in all samples [2]. In terms of grain orientation, the annealing treatment promotes the formation of [100] texture in the $30 \mathrm{~nm}$ films, and a near [111] texture for the $120 \mathrm{~nm}$ film (Fig. 2). In regards to grain size, the annealing treatment leads to grain growth in all samples, although pockets of very small grains remain stable and do not grow (Fig. 3).

\section{References}

[1]A. Kobler et al. Ultramicroscopy, 128 (2013), p.68-81

[2] S. Rajasekhara et al. Scripta Materialia, 67. (2012), p.189-192

[3] Acknowledgments: This work was fully supported by the Division of Materials Science and Engineering, Office of Basic Energy Sciences, US Department of Energy. Sandia National Laboratories is a multi-program laboratory operated by Sandia Corporation, a wholly owned subsidiary of Lockheed Martin Company, for the US Department of Energy's National Nuclear Security Administration under contract DE-AC04-94AL85000. 
(a)

(b)
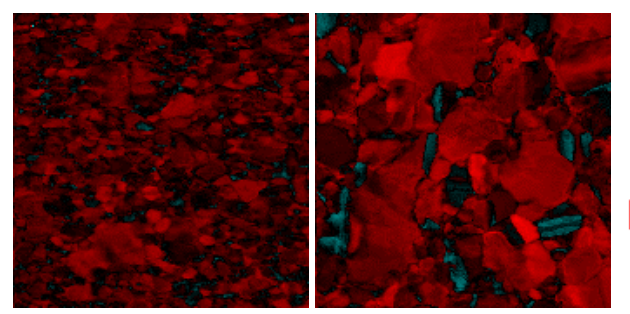

FCC Phase

HCP Phase

Fig. 1 Phase maps combined with reliability maps for samples of thickness (a) $30 \mathrm{~nm}$ and (b) $120 \mathrm{~nm}$. The presence of fcc and hcp phases in both samples is evident.

(a)

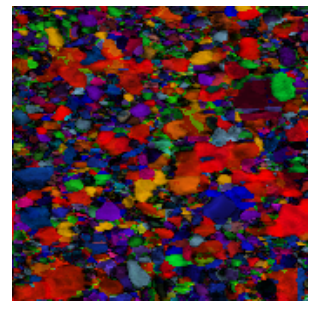

(e)

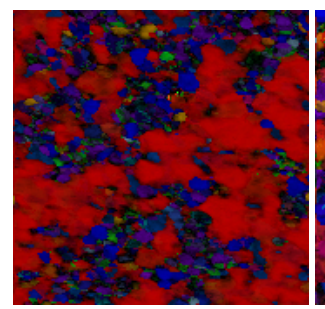

(b)

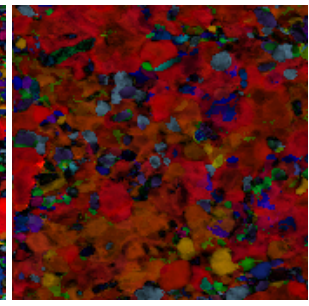

(f)

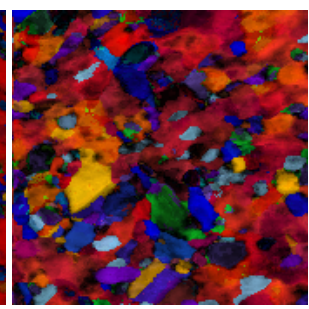

(c)

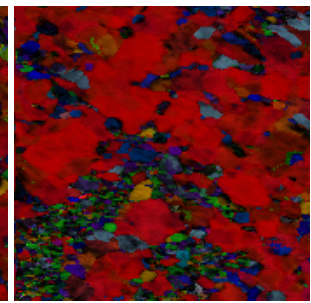

(g)

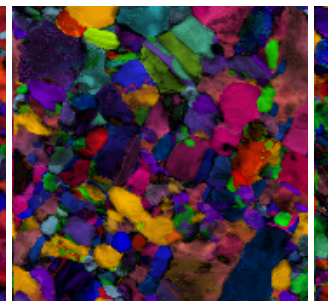

(d)

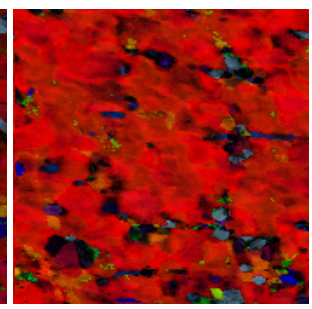

(h)

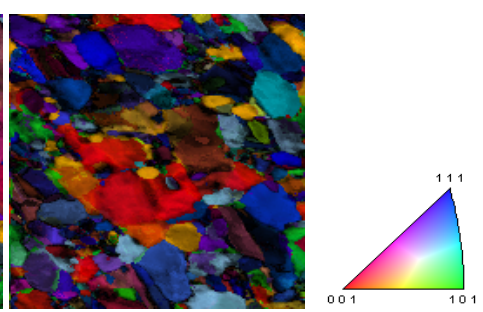

Fig. 2 Orientation maps combined with reliability maps for 30nm films annealed for (a) 20min, (b) $40 \mathrm{~min}$, (c) $60 \mathrm{~min}$ and (d) $80 \mathrm{~min}$ and $120 \mathrm{~nm}$ film annealed for (e) $20 \mathrm{~min}$ (f) $40 \mathrm{~min},(\mathrm{~g}) 60 \mathrm{~min}$ and (h) $80 \mathrm{~min}$.

(a)

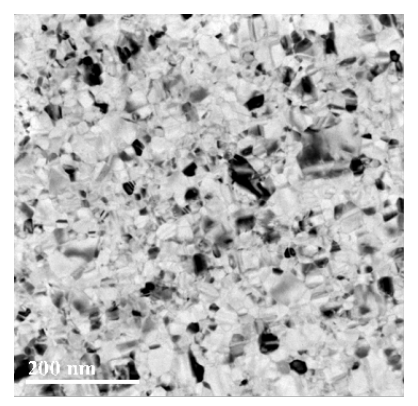

(b)

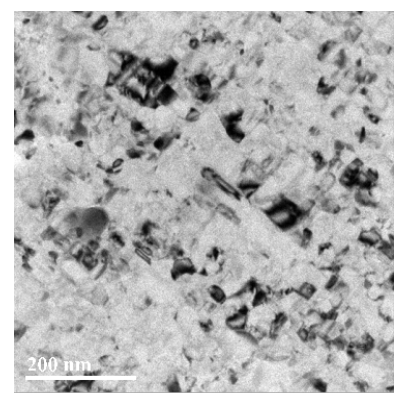

Fig. 3 TEM bright field images showing grain growth and pockets of small grains in the $30 \mathrm{~nm}$ thin films after annealing for (a) $20 \mathrm{~min}$ and (b) $60 \mathrm{~min}$. 